\title{
EL SENTIDO DE LA INVESTIGACIÓN CUALITATIVA
}

\section{Almudena Cotán Fernández}

\section{RESUMEN}

La investigación cualitativa surge como paradigma alternativo al paradigma racionalista puesto que entre las diferentes disciplinas existentes en el ámbito social existen variopintas problemáticas, interrogantes, cuestiones y restricciones que no se pueden abordar en su extensión a través de la metodología cuantitativa, por lo que, surge una nueva metodología procedente de la antropología, la sociología y la etnografía, entre otros.

El presente trabajo presenta una revisión teórica sobre el método de investigación cualitativo desde su origen a mediados del siglo $X I X$, principios del $X X$, donde sus esfuerzos se diriǵan a demostrar su validez y rigurosidad en la interpretación de sus datos, hasta la actualidad, con un panorama muy diferente donde no se cuestiona su validez, pasando a convertirse en uno de los métodos más empleados dentro de las Ciencias Sociales.

Palabras clave: metodología cualitativa, ciencias sociales, significatividad, experiencias, participación.

\section{TITLE: THE SENSE OF QUALITATIVE INVESTIGATION}

\section{ABSTRACT}

Qualitative investigation emerged as an alternative to the rationalist paradigm since between the different existing disciplines in the social field there are a series of problems, interrogations, questions and restrictions which cannot be tackled in every sense through quantitative methodology, therefore, a new methodology emerges from anthropology, sociology and ethnography, among others.

This paper presents a theoretical revision on the qualitative method of investigation since its origin in the middle of the 19th century, beginning of the 20th, where its efforts were directed towards demonstrating its validity and rigorousness in the interpretation of data, up until the present day, with a very different panorama where its validity is not questioned, and becoming one of the most used methods within Social Sciences.

Keywords: Qualitative Methodology, Social Sciences, Meaningfulness, Experiences, Participation.

Correspondencia con la autora: Almudena Cotán Fernández. Departamento de Educación. Universidad Isabel I. <almudena.cotán@uil.es>.0riginal recibido:01-02-15.0riginal aceptado: 19-03-16. 


\section{Las primeras apariciones de la metodología cualitativa}

Podemos hallar el origen de la investigación cualitativa a finales del siglo XIX cuando los antropólogos y sociólogos empiezan a darle importancia a los datos recogidos en el campo de investigación y no en los laboratorios; cuando la investigación naturalista pone al investigador en el contexto natural donde acontece el suceso que va a ser estudiado y cuando la etnografía empieza a cobrar importancia, involucrando e integrando al investigador o investigadora en la comunidad que va a estudiar, quedando la objetividad relegada a un ejercicio subjetivo de la comprensión (Amezcua y Gálvez, 2002; Cortés, 2013; Flick, 2007; Rodríguez, Gil y García, 1996; Ruiz Olabuénaga, 20 I 2; Sandín, 2003). Según Flick (2007) y Delgado y Gutiérrez (I999), la importancia de la investigación radica en el estudio de las relaciones sociales.

La utilización de la metodología cualitativa cuenta con una larga historia en la psicología y las ciencias sociales (Flick, 2007; Ruiz Olabuénaga, 20I2). Si seguimos a Bogdan y Biklen (1982), ellos sitúan el origen de esta metodología en la reciente historia de Estados Unidos, cuyos movimientos de urbanización de inmigración masiva provocaron problemas de índole sanitario, educativo, laborales, etc. (Rodríguez, Gil y García, 1996; Sandín, 2003).

No obstante, cuando hablamos de investigación cualitativa no podemos olvidarnos de su perspectiva fenomenológica considerándose el origen de la misma, centrando sus planteamientos en el estudio de la conducta humana como marco de referencia, "el fenomenólogo, intenta ver las cosas desde el punto de vista de otras personas" (Taylor y Bogdan, 2010, p. 23).

En la actualidad, la investigación cualitativa ha dado un giro importante permitiendo y fomentando la participación de las personas en la investigaciones, dando lugar, por lo tanto, a investigaciones más participativas y democráticas donde se tenga en cuenta las perspectivas y voces oprimidas y silenciadas (Cortés, 2013; De la Rosa, 2008; Goodley et al., 2004; Lincoln y Denzin, 1994; Moriña, 2003).

Entre las características principales de la investigación cualitativa, se puede mencionar el emplazamiento natural de la misma, la significatividad de las perspectivas de las historias narradas y su comprensión (Parrilla, 2000), así como el énfasis en comprender el proceso en el que se ha llevado a cabo las historias, comprendiendo los roles, las políticas sociales, etc. (Ameigeiras, 2006; Flick, 2007; Moriña, 2003; Parrilla, 2000; Pujadas, 2002; Sandín, 2003; Taylor y Bogdan, 2010; Vallés, 1999). 
Para Taylor y Bogdan (2010), la metodología cualitativa se caracteriza por ser inductiva, holística, humanista así como por el papel que juegan los investigadores:

a) son sensibles a los efectos que ellos mismos causan sobre la persona; b) se centran en comprender a la persona en su contexto; c) deben de suprimir o separar sus propias creencias, perspectivas y predisposiciones; d) deben de aceptar todas las perspectivas como valiosas $y$, e) dan énfasis a la validez de su propia investigación.

Desde un carácter interpretativo, Eisner (1998) plantea dos enfoques de la metodología cualitativa:

- Justificación, elaboración e integración en un marco teórico los datos obtenidos, $y$,

- Dar voz a los propios participantes de la investgación, acercándonos a sus experiencias particulares desde su significado y visión de los hechos.

Para Parrilla (2000), la investigación cualitativa tiene gran importancia debido a la experiencia subjetiva de los individuos en la construcción del mundo social, concibiendo la realidad como múltiple y divergente. Por ello, el compromiso en esta metodología es estudiar y analizar el mundo desde la perspectiva de los participantes (Ameigeiras, 2006; Moriña, 2003; Parrilla, 2000; Pujadas, 1992, 2002; Sandín, 2003; Taylor y Bogdan, 20 I0; Vallés, 1999).

Taylory Bogdan (2010), hablan de la existencia de dos enfoques teóricos principales en la metodología cualitativa que merecen ser reseñados: el interaccionismo simbólico y la etnometodología.

El interaccionismo simbólico, centra sus estudios en los significados sociales que las personas otorgan a su contexto; por su parte, la etnometodología, integrada dentro del interaccionismo simbólico, estudia la forma en que las personas aplican las reglas y normas culturales y sociales a actuaciones concretas y rutinarias, es decir, el interaccionismo simbólico estudia la realidad y la etnometodología estudia la realidad de la vida cotidiana.

Sin embargo y de acuerdo con Moriña (2003), la evolución por la que ha pasado la metodología cualitativa se encuentra llena de rupturas y de grandes dosis de “incertidumbre, contradicción e inseguridad” (Moriña, 2003, p. 34I), por lo que, al posicionarnos ante esta metodología asumimos que no existe un único camino de hacer investigación cualitativa, sino múltiples, además de diferentes enfoques y 
perspectivas que potencian y estimulan la creatividad, implicación personal, reflexión conjunta y apertura mental y emocional (Moriña, 2003; Parrilla, 2000).

A diferencia de la evolución más paulatina que han podido tener otro tipo de metodologías o investigaciónes como, por ejemplo, la cuantitativa, la investigación cualitativa ha tenido un importante proceso de evolución creciente que abarca desde las críticas realizadas hacia su cientificidad y rigurosidad, pasando por la pluralidad de estilos narrativos hasta llegar a la actualidad donde el investigador se suele centrar en dar respuesta a las cuestiones de investigación planteadas a partir de las experiencias reales y subjetivas dándole voz a los participantes para que éstos puedan ser escuchados a través de sus experiencias (De La Rosa, 2008; Eisner, 1998; Flick, 2007; Goodley et al., 2004; Lincoln y Denzin, 1994; Moriña, 2003; Parrilla, 2000; Taylor y Bogdan, 2010).

No obstante, dentro de la evolución anteriormente reseñada, sería importante indicar de forma escueta las etapas por las que la investigación cualitativa ha pasado, más señalado por las rupturas que por un progresivo movimiento de un estado a otro (Moriña, 2003) (Ver tabla I).

En un primer momento, destacado por autores como Denzin y Lincoln (1998), los inicios del siglo $X X$ (1900), se caracterizan por los esfuerzos de la metodología cualitativa por demostrar su validez y rigurosidad en la interpretación de sus datos. Es el momento de la etnografía clásica (Vallés, 1999) y, en especial, del etnógrafo solitario en el área de la antropología. Holstein y Gubrium (20I2) consideran que a través de las acciones que los sociólogos pertenecientes a la escuela de Chicago, coleccionando historias de vida y guardando documentos personales durante los años 20 hasta los 30 , se anticiparon a lo que hoy en día consideramos como investigación narrativa.

En un segundo momento (1959-1970), se caracteriza por un mayor asentamiento y se empiezan a encontrar informes descriptivos altamente elaborados (Moriña, 2003). En este período modernista, se empiezan a realizar varios esfuerzos de formalización de los métodos cualitativos (Glaser y Strauss, 1967; Moriña, 2003; Taylor y Bogdan, 2010). 


\begin{tabular}{|c|c|c|}
\hline $\begin{array}{l}\text { Paréntesis } \\
\text { cronológico }\end{array}$ & $\begin{array}{l}\text { Denominación del } \\
\text { período histórico }\end{array}$ & Caracterización \\
\hline$(1900-1959)$ & Tradicional & $\begin{array}{l}\text { Positivismo. Época del etnógrafo } \\
\text { solitario. }\end{array}$ \\
\hline$(1959-1970)$ & $\begin{array}{l}\text { Modernista o "edad } \\
\text { dorada" }\end{array}$ & $\begin{array}{l}\text { Postpositivismo. Análisis cualitativo } \\
\text { riguroso. }\end{array}$ \\
\hline (1970-1986) & $\begin{array}{l}\text { Géneros } \\
\text { desdibujados }\end{array}$ & Interpretativo. Geertz (1973, 1983). \\
\hline$(1986-1990)$ & $\begin{array}{l}\text { Crisis de } \\
\text { representación }\end{array}$ & $\begin{array}{l}\text { Reflexividad, (auto) crítica. Marcus y } \\
\text { Fischer (1986), Clifford (1988). }\end{array}$ \\
\hline (I990-Actualidad) & Postmoderno & $\begin{array}{l}\text { Descubrimiento y redescubrimiento } \\
\text { de modos de investigar cualitativos. }\end{array}$ \\
\hline
\end{tabular}

Tabla I. Momentos en la investigación cualitativa

(Vallés, 1999, p.32, basado en Denzin y Lincoln, 1998).

En un tercer momento, denominado como el momento de los "géneros desdibujados" o "borrosos" (Denzin y Lincoln, 1998), en torno a los años 70-80, la metodología cualitativa empieza a ganar terreno y a afianzarse desplegando ya un amplio abanico de paradigmas (post-estructuralismo, neopositivismo, neo-marxismo, deconstruccionismo, etnometodología, etc.; Vallés, 1999), métodos, estrategias y formas de recogida y análisis de la información. Tal y como la denominan Denzin y Lincoln (1998) la "edad dorada" de la investigación cualitativa. Se puede situar al antropólogo Geertz (1973, 1983) como el autor que abre y cierra ésta época con sus obras The Interpretation of Cultures (1973) y Local Knowledge (1983). Este autor nos lleva a reflexionar sobre las formas de presentación y de representación de los textos así como la legitimación de las etnografías (Vallés, 1999).

A mediados de la década de los 80 e inicio de los años 90, surge la "crisis de la representación" (Denzin y Lincoln, 1998), derivada de la fase anterior, con un proceso de maduración de la misma. En la crisis de la representación, se establece una dicotomía de polos enfrentados, por un lado, se establece la representación de 
los investigadores en los textos escritos poniéndose en duda que éstos hagan acopio directo de la experiencia vivida (Denzin y Lincoln, 1994) y, por otro, la crisis de la legitimación, expone que se haga una revisión de los criterios para evaluar los diseños y los resultados de la investigación cualitativa establecidos hasta el momento, ya que se consideran caducos. Adquieren bastante importancia los conceptos de validez y fiabilidad (Vallés, 1999).

Los temas relacionados con la política y la ética en investigación cualitativa empiezan a adquirir interés para la comunidad científica del momento (Sandín, 2003). Y por último, el quinto momento denominado Postmoderno (1990-hasta la actualidad), ya no se cuestiona la validez del método cualitativo y se empiezan a convertir las ciencias sociales en un lugar donde "se insertan conversaciones críticas acerca de la democracia, raza, género, nación, libertad y comunidad” (Sandín, 2003, p.84).

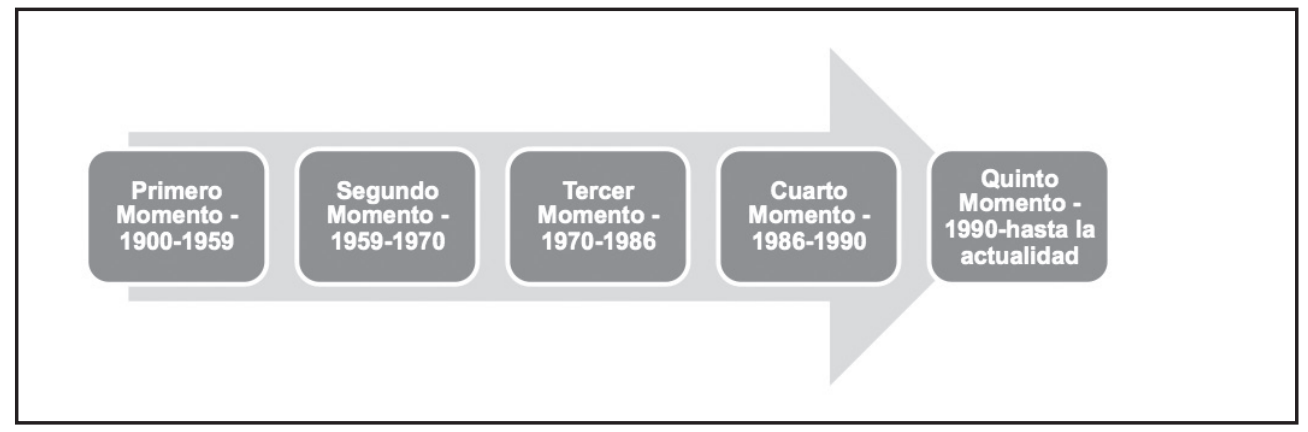

Figura I. Momentos en la Investigación Cualitativa (Fuente: elaboración propia).

No obstante, estamos de acuerdo con Denzin y Lincoln (1998) y Moriña (2003) al indicar que detrás de estas cinco etapas en la investigación cualitativa (véase figura I), reconocemos que todas ellas continúan actuando en el presente y es por ello, que este tipo de metodología se caracteriza por una amplia variedad de elecciones de investigar que nos permiten nuevas formas de mirar, interpretar, argumentar y escribir. Tal y como indica Vallés (1999), no existe un modelo único de investigación ni un modelo que podamos emplear en dos investigaciones seguidas, cada diseño tiene su propia casuística, variables, necesidades y problemas, entre otros. Sin embargo, 
a diferencia de otro tipo de metodologías, la cualitativa no puede verse desde una perspectiva positivista, neutral u objetiva, ya que se encuentra fuertemente cargada de subjetividades tanto del investigador como de los participantes que comparten el proceso de la investigación.

\section{Características de la investigación cualitativa}

A lo largo de la historia y la literatura escrita sobre investigación cualitativa, han sido muchos los autores que han hablado sobre sus características (Denzin y Lincol, 1998; Hammersley y Atkinson, 1994; Minichiello, Aroni, Timewell y Alexander, 1990; Moriña, 2003; Parrilla, 2000; Rodríguez, Gil y García, 1996; Sandín, 2003; Simons, 1996; Taylor y Bogdan, 20 I0; Vasilachis, 2006). Sin embargo, para no ser redundantes ni repetitivos al enunciarlas de nuevo, ya que no es el objetivo de este apartado reiterar a estos autores, nos centraremos en delimitar y seleccionar aquellas características que consideremos relevantes y significativas para nuestro trabajo.

En primer lugar, entre las principales características de la investigación cualitativa, podemos mencionar el emplazamiento natural de la misma, la significatividad y perspectivas de las historias narradas así como su comprensión (Parrilla, 2000) y el énfasis en comprender el proceso en el que se ha llevado a cabo las historias, comprendiendo los roles, las políticas sociales, etc. (Ameigeiras, 2006; Flick, 2007; Sandín, 2003; Parrilla, 2000; Pujadas, 2002; Taylor y Bogdan, 20 I0; Vallés, 1999).

Desde su origen, la investigación cualitativa se caracteriza por desplazarse al contexto natural donde acontecen las experiencias, donde viven las personas y donde puede recoger las situaciones, es decir, el investigador se centra en recoger todos los momentos de las situaciones vividas por los participantes dentro de su emplazamiento natural. Pero para ello, el investigador debe de mantener apertura mental sin prejuzgar ni conformarse con las primeras apariencias.

Respecto a la significatividad, perspectivas y comprensión de las historias narradas, el investigador ha de descubrir e identificar el significado que los participantes atribuyen a los acontecimientos y conductas, cómo interpretan las situaciones y cuáles son sus perspectivas personales sobre las mismas. Para ello, Moriña (2003) y Simons (1996), indican que para facilitar dicho proceso el investigador ha de describir e interpretar los acontemientos liberando la mente de cualquier tipo de concepto 
o indicador preconcebido, con el fin de percibir y comprender lo que se quiere investigar desde su esencia (Sandoval, 1996).

Complementando la caracterización anterior, debemos mencionar autores como Taylor y Bogdan (20I0), quienes señalan los siguientes rasgos como hitos principales en la investigación cualitativa:

I. Es inductiva: se centra en descrubir y hallar más que en comprobar o verificar.

2. Es holística: el investigador ve a las personas y a los grupos como un todo que engloba los procesos de organización, funcionamiento y significados. No son fragmentados en variables.

3. Es interactiva y reflexiva: los investigadores son sensibles a los efectos que causan sobre los participantes de su estudio (Sandoval, 1996).

4. Es naturalista: tal y como hemos indicado, los investigadores se centran en conocer a las personas y situaciones que le acontecen en su contexto natural.

5. No tiene ideas previas ni preconcebidas.

6. Es abierta: no excluye escenarios ni visiones en la recolección de datos. Todas las perspectivas son válidas.

7. Es humanista: abarca el lado privado y personal de las experiencias centrándose en las percepciones, concepciones y significados de los protagonistas.

Por su parte, Parrilla (2000) opina que en la investigación cualitativa tiene gran importancia la experiencia subjetiva de los individuos en la construcción del mundo social, concibiendo la realidad como múltiple y divergente. Por ello, esta metodología está comprometida con estudiar y analizar el mundo desde la perspectiva de los participantes (Ameigeiras, 2006; Parrilla, 2000; Pujadas, 2002; Sandín, 2003; Taylor y Bogdan, 2010; Vallés, 1999). Por ello, Miles y Huberman (1994), consideran que la investigación cualitativa debe de realizarse a través de un prolongado e intenso contacto con el campo de estudio y los protagonistas, donde el investigador tiene que alcanzar una visión holística capturando las percepciones de los actores a través de un proceso de comprensión empátiva y de suspensión o ruptura de las preconcepciones previas (Rodríguez, Gil, y García, 1996). 
Para Flick (2007) y Vasilachis (2006), la investigación cualitativa busca teorías empíricas posicionándose desde una perspectiva de comprensión, construyendo y comprendiendo la influencia que ejercen unos sucesos en otros a través de una red de relaciones causales, locales, contextuales y situacionales.

Siguiendo a Rodríguez, Gil y García (1996, p. 56), podemos decir que la investigación cualitativa tiene cuatro ventajas fundamentales:

a) Se combina la participación con la investigación, superando por lo tanto los procedimientos tradicionales de conocimiento llegando a la unión entre la teoría y la práctica.

b) Se acentúa el compromiso político desde una posición crítica emancipadora.

c) Se potencia el carácter educativo de la investigación y,

d) La necesidad de devolver lo investigado a la población como medio de empoderamiento.

Por lo que, estamos de acuerdo que al posicionarnos ante una investigación de índole cualitativo asumimos los profundos procesos de reflexión e implicación que tenemos que ejercer, manteniendo una relación constante e interactiva con los principales actores de la investigación para poder obtener un proceso de recolección e interpretación de los datos que nos permita conocer la realidad que estamos estudiando. Para ello, Moriña (2003) indica que debemos mantener un énfasis en el proyecto interesándonos por conocer cómo se forman las comprensiones, significados, roles, etc. Por lo que no tenemos más remedio que acudir a lo que Denzin y Lincoln (1998) denominan como “descripción densa", donde los investigadores ahondan en el significado que los actores atribuyen a los acontecimientos.

\section{3. ¿Cómo se concreta la investigación cualitativa? Métodos}

Dado el abundante material consultado al respecto, no queremos finalizar este trabajo sin antes realizar una breve revisión sobre los métodos cualitativos existentes. No obstante, debemos indicar que no es nuestra pretensión realizar una revisión exhaustiva sobre los diferentes métodos o estrategias cualitativas existentes puesto que consideramos que existe bibliografía que hace una revisión completa (véase el trabajo de Rodríguez, Gil, y García, 1996 o Sandoval, 1996). 
42 - El sentido de la investigación cualitativa

Qualitative Strategies in Educational Research (Wolcott, 1992)

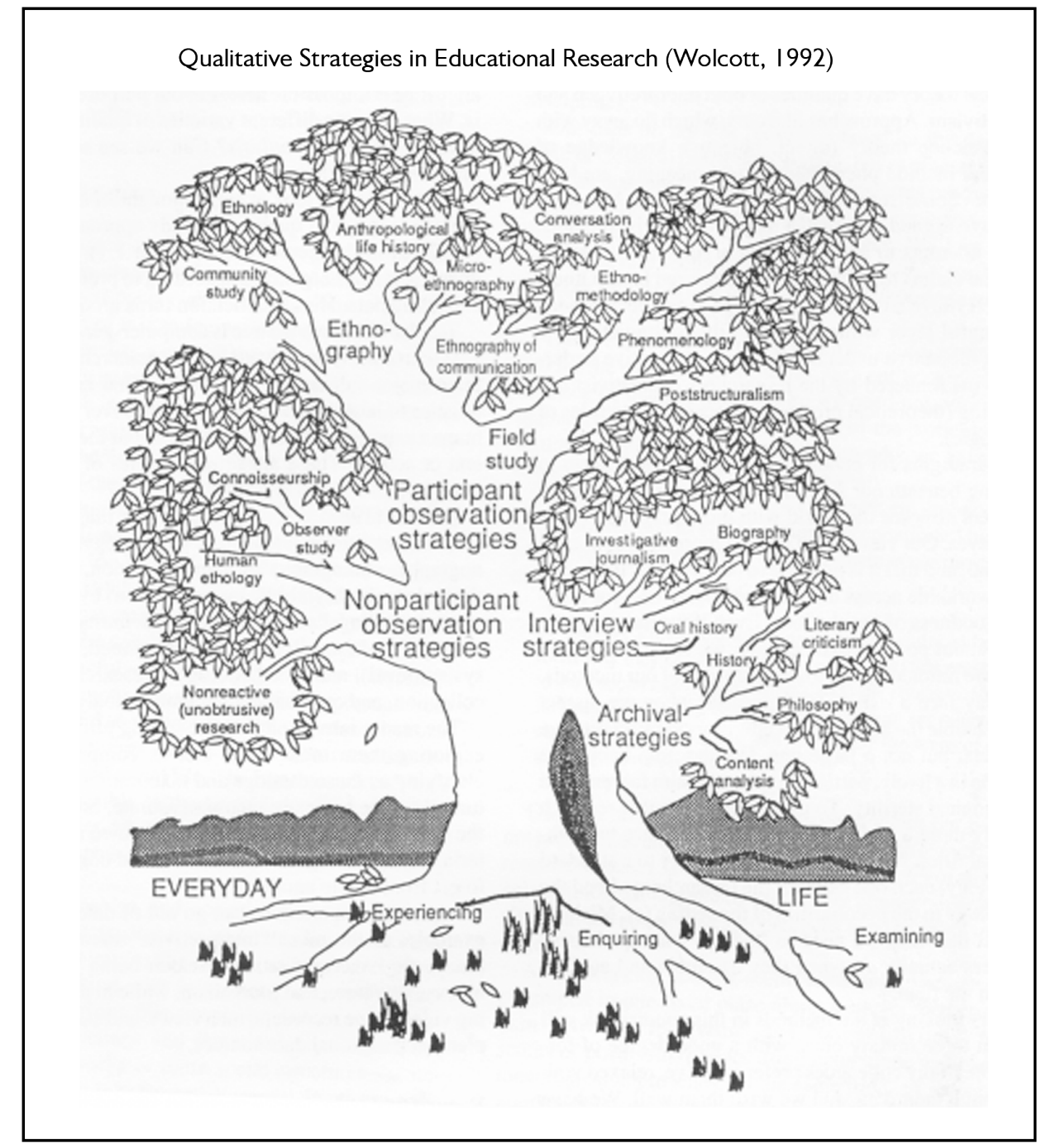

Figura 2. Estrategias educativas en investigación cualitativa (Wolcott, 1992, p. 23). 
Tras la lectura de varios documentos (Axpe, 2003; Rodríguez, Gil y García, 1996; Sandelowski, 2000), vimos como hacían alusión al árbol de Wolcott (1992) para representar las distintas estrategias cualitativas existentes (ver figura 2). Para ello, este árbol parte de la experiencia diaria; en sus raíces podemos leer tres cuestiones esenciales en este proceso indagador: experimentar, preguntar y examinar. A partir de éstas, desarrolla todo un entramado de ramas y hojas que representa los diferentes modelos y estrategias que la investigación cualitativa ofrece al investigador para poder trabajar. Tal y como indica Axpe (2003, p. 29), "el árbol representa las interrelaciones de los enfoques cualitaivos. El tronco es el centro común de las técnicas de investigación. Posteriormente, éste se va dividiendo en miembros fuertes y después en pequeñas ramificaciones".

Otros trabajos como los de Rodríguez, Gil y García (1996) o Sandoval (1996), recogen las estrategias cualitativas existentes. A diferencia de Sandoval (1996) quien realiza un mapa conceptual con los diferentes métodos y estrategias que existen, Rodríguez, Gil y García (1996), realizan un cuadro comparativo donde especifican los métodos, fuentes, técnicas de recogida de información y principales referentes de las mismas. Desde nuestro punto de vista y sin menospreciar el excelente resumen realizado por Sandoval (1996), en este apartado vamos a decantarnos por la clasificación realizada por Rodríguez, Gil y García (1996) puesto que nos parece muy clarificadora (ver tabla 2).

Como podemos observar en el cuadro, el primero de los métodos que aparece es la fenomenología a principios del siglo $X X$, que destaca el énfasis en lo individual y en las experiencias. Continuando con el cuadro, el método etnográfico, se centra en la forma de vida como unidad social. La teoría fundamentada con profundas implicaciones del interaccionismo simbólico, se centra en determinar el significado de los gestos y palabras que son atribuidos por los grupos sociales y la forma de interactuar entre los mismos. El cuarto método, la etnometodología, situada a finales del siglo XX (años 60-70), estudia los fenómenos sociales asociados a nuestra actividad humana en situaciones cotidianas. Finalizando ya con el cuadro, nos encontramos con los dos últimos métodos: investigación-acción y la biografía. La investigación-acción destaca por el carácter activo que los participantes de la investigación adquieren durante la investigación que parte de problemas surgidos de la práctica educativa, 
reflexionando sobre los mismos y rompiendo la dicotomía existente entre la teoría y la práctica. Dentro de ésta podemos encontrar la investigación-acción del profesor, investigación-acción, la investigación cooperativa y la investigación participativa. Por otro lado, la biografía, refleja el testimonio de una persona recogiendo situaciones, experiencias y acontecimientos desde un punto de vista subjetivo, dando forma a una historia de vida o relato autobiográfico.

\begin{tabular}{|c|c|c|c|c|}
\hline $\begin{array}{c}\text { Tipos de } \\
\text { cuestiones de } \\
\text { investigación }\end{array}$ & Método & Fuentes & $\begin{array}{c}\text { Técnicas / } \\
\text { instrumentos } \\
\text { de recogida de } \\
\text { información }\end{array}$ & $\begin{array}{c}\text { Otras fuentes } \\
\text { de datos }\end{array}$ \\
\hline $\begin{array}{l}\text { Cuestiones de } \\
\text { significado: Explicitar } \\
\text { la esencia de la } \\
\text { experiencia de los } \\
\text { actores }\end{array}$ & Fenomenología & $\begin{array}{c}\text { Filosofía } \\
\text { (fenomenología) }\end{array}$ & $\begin{array}{l}\text { Grabación de } \\
\text { conversaciones } \\
\text { Escribir } \\
\text { anécdotas de } \\
\text { experiencias } \\
\text { personales }\end{array}$ & $\begin{array}{l}\text { Literatura } \\
\text { fenomenológica. } \\
\text { Reflexiones } \\
\text { filosóficas; } \\
\text { poesía, arte }\end{array}$ \\
\hline $\begin{array}{l}\text { Cuestiones } \\
\text { descriptivas- } \\
\text { interpretativas: } \\
\text { valores, ideas, } \\
\text { prácticas de los } \\
\text { grupos culturales }\end{array}$ & Etnografía & Antropología & $\begin{array}{c}\text { Entrevista no } \\
\text { estructurada; } \\
\text { observación } \\
\text { participantes, } \\
\text { diagrama de } \\
\text { redes sociales }\end{array}$ & $\begin{array}{l}\text { Documentos, } \\
\text { registros, } \\
\text { fotografía, } \\
\text { mapas, } \\
\text { genealogía, } \\
\text { diagrama de } \\
\text { redes sociales }\end{array}$ \\
\hline $\begin{array}{l}\text { Cuestiones de } \\
\text { proceso: experiencia } \\
\text { a lo largo del tiempo } \\
\text { o el cambio puede } \\
\text { tener etapa u fases }\end{array}$ & $\begin{array}{c}\text { Teoría } \\
\text { fundamentada }\end{array}$ & $\begin{array}{c}\text { Sociología } \\
\text { (interaccionismo } \\
\text { simbólico) }\end{array}$ & $\begin{array}{l}\text { Entrevistas } \\
\text { (registradas en } \\
\text { cintas) }\end{array}$ & $\begin{array}{c}\text { Observación } \\
\text { participante; } \\
\text { memorias, } \\
\text { diarios. }\end{array}$ \\
\hline $\begin{array}{c}\text { Cuestiones } \\
\text { centradas en la } \\
\text { interacción y el } \\
\text { diálogo }\end{array}$ & $\begin{array}{l}\text { Etnometodología: } \\
\text { análisis del } \\
\text { discurso }\end{array}$ & Semiótica & $\begin{array}{c}\text { Diálogo } \\
\text { (registro en } \\
\text { audio y video) }\end{array}$ & $\begin{array}{l}\text { Observación: } \\
\text { notas de campo }\end{array}$ \\
\hline $\begin{array}{l}\text { Cuestiones de } \\
\text { mejora y cambio } \\
\text { social }\end{array}$ & $\begin{array}{l}\text { Investigación- } \\
\text { acción }\end{array}$ & Teoría crítica & Miscelánea & Varios \\
\hline $\begin{array}{l}\text { Cuestiones } \\
\text { subjetivas }\end{array}$ & Biografía & Antropología & Entrevista & $\begin{array}{l}\text { Documentos, } \\
\text { registros, diarios }\end{array}$ \\
\hline
\end{tabular}

Tabla 2. Métodos cualitativos (Rodríguez, Gil y García, 1996, p. 18). 


\section{Conclusiones}

En la actualidad, la investigación cualitativa ha dado un giro importante permitiendo y fomentando la participación de las personas en la investigaciones, dando lugar, por lo tanto, a investigaciones más participativas y democráticas donde se tiene en cuenta las perspectivas y voces oprimidas y silenciadas (Booth, 1996; Cortés, 2013; De la Rosa, 2008; Goodley et al., 2004; Lincoln y Denzin, 1994). Pensamos que el conocimiento pedagógico, dependiente de las realidades humanas, tiene como objetivo construir interpretaciones desde las percepciones y experiencias de los participantes.

Por lo tanto, podemos concluir el presente trabajo afirmando que en la metodología cualitativa, el investigador tiene que centrarse en dar respuesta a las cuestiones de investigación planteadas a partir de las experiencias reales de las personas. El fin de esta metodología no es otro que dar voz a los participantes para que éstas sean escuchados a través de sus experiencias, comunicadas de manera abierta.

\section{Referencias bibliográficas}

Ameigeiras, A. R. (2006). El abordaje etnográfico en la investigación social. En I. Vasilachis de Gialdino, (Coord.), Estrategias de Investigación Cualitativa (pp. I07I5I). Barcelona: Gedisa.

Amezcua, M. y Gálvez, A. (2002). Los modos de análisis en investigación cualitativa en salud: perspectiva crítica y reflexiones en voz alta. Revista Española Salud Pública, 76, 423-436.

Axpe, M. A. (2003). La investigación etnográfica en el campo de la educación. Una aproximación meta-analítica. Tesis Doctoral. Universidad de la Laguna, Santa Cruz de Tenerife, España.

Bisquerra, R. (Coord.) (2004). Metodología de la investigación educativa. Madrid: Editorial la Muralla. 
Bogdan, R. C. y Biklen, S. K. (1982). Qualitative Research for Education: an introduction to theory and methods. London: Allyn and Bacon.

Booth, T. (1996). A Perspective on Inclusion from England. Cambridge Journal of Education, 26 (I), 87-99.

Cortés, P. (20I3). El guiño del poder, la sonrisa del cambio. Estudio pedagógico sobre Identidad Resiliente en situaciones de desventaja social, cultural y jurídica. Tesis Doctoral, Facultad Ciencias de la Educación, Universidad de Málaga, Málaga, España.

De La Rosa, L. (2008). La historia de vida de Ángel. Parálisis cerebral, normalidad y comunicación. Madrid: La Muralla.

Delgado, J. M. y Gutiérrez, J. (1999). Métodos y técnicas cualitativas de investigación en ciencias sociales. Madrid: Síntesis.

Denzin, N. K., y Lincoln, Y. S. (1998). The landscape of qualitative research. New Delhi: Sage Publications.

Eisner, E. (1998). El ojo ilustrado. Indagación cualitativa y mejora de la práctica educativa. Barcelona: Paidós.

Flick, U. (2007). Introducción a la Investigación Cualitativa. Madrid: Morata.

Geertz, C. (1973). The interpretation of cultures. New York: Basic Books.

Geertz, C. (1983). Local knowledge. New York: Basic Books.

Glaser, B. y Strauss, A. (1967). The discovery of grounded theory: strategies for qualitative research. New York: Aldine Publishing Company.

Goodley, D., Lawthom, R., Clough, P. y Moore, M. (2004). Researching life stories. London: Routledge.

Hammersley, M. y Atkinson, P. (1994). Etnografía. Métodos de investigación. Barcelona: Paidós. 
Holstein, J. y Gubrium, J. (Eds). (20I2). Varieties of Narrative Analysis. Thousand: Oaks, Sage.

Lincoln, Y. y Denzin, N. (1994). The Fith Moment. En N. Denzin y. Lincoln (Eds.), Handbook of Qualitative Research (pp. 575-586). London: Sage Publications.

Marcus, G. y Fischer, M. (1986). Anthropology as Cultural Critique. Chicago: University of Chicago Press.

Miles, M. B. y Huberman, A. M. (1994). Qualitative data analysis. Beberly Hills, CA: Sage Publications.

Minichiello, V., Aroni, R., Timewell, E. y Alexander, L. (1990). In-depth interviewing. Melbourne: Longman.

Moriña, A. (2003). Diversidad en la escuela: Diseño, Desarrollo y Valoración de una propuesta de formación para el profesorado. Tesis Doctoral, Tomo I, Universidad de Sevilla.

Parrilla, A. (2000). Proyecto docente e investigador II. Cátedra de Universidad, Universidad de Sevilla.

Pérez, G. (1994). Investigación Cualitativa. Retos e interrogantes. I Métodos. Madrid: Editorial la Muralla.

Pujadas, J. J. (2002). El método biográfico: El uso de las Historias de Vida en Ciencias Sociales. Madrid: Centro de Investigaciones Sociológicas (edición original, 1992).

Rodríguez, G., Gil, J. y García, E. (1996). Metodología de la Investigación Cualitativa. Granada: Aljibe.

Ruiz Olabuénaga, J. L. (20I2). Metodología de la investigación cualitativa. Bilbao: Universidad de Deusto.

Sandelowski, M. (2000). Whatever happened to qualitative description? Research in Nursing \& Health, 23, 334-340. 
Sandín, M. P. (2003). Investigación Cualitativa en Educación. Fundamentos y Tradiciones. Madrid: McGraw Hill.

Sandoval, C. (1996). La formulación y el diseño de los procesos de investigación social cualitativos. En C. Sandoval, Investigación cualitativa (pp. I I I-I28). Bogotá: Instituto Colombiano para el Fomento de Educación Superior.

Simons, D. J. (1996). In sight, out of mind: When object representations fail. Psychological Science, 7, 30I-305.

Taylor, S. J. y Bogdan, R. (2010). Introducción a los métodos cualitativos. Nueva York: Book Print (edición original, 1992).

Vallés, M. S. (1999). Técnicas Cualitativas de Investigación Social. Madrid: Síntesis.

Vasilachis de Gialdino, I. (Coord.). (2006). Estrategias de Investigación Cualitativa. Barcelona: Gedisa.

Wolcott, H. F. (1992). Posturing in qualitative research. En M. LeCompte, W Millroy, y J. Preissle (Eds.), The Handbook of Qualitative research in education (pp. 3-52). London: Academic Press. 